\title{
The Global Response to COVID-19 as an Example of a One-Sided Problem Definition in the Absence of General Collective Intelligence
}

Andy E. Williams

Nobeah Foundation, Nairobi, Kenya

Email: awilliams@nobeahfoundation.org

\begin{abstract}
Problem definitions are defined here as one-sided in the case that while they might take into account one class of negative outcomes, such as those associated with the problem, at the same time they might ignore other classes of negative outcomes, such as those that may be encountered while implementing interventions that try to avoid the problem. An example is amputating all limbs with potentially cancerous moles on them to reduce the risk of mortality due to cancer as much as possible, without considering the increase in mortality due to the amputations. The global response to COVID-19 has been characterized by the availability of mathematical models for the potential mortality due to the spread of the pandemic. However in some cases the researchers guiding the responses of their respective nations with their mathematical models have explicitly pointed out that corresponding mathematical models of the impacts of economic shutdowns or other potential interventions on mortality have not been incorporated, and that there is a critical need to include such models. This paper generalizes this problem of one-sided problem definitions past the COVID-19 response to a wide variety of group problems where the pattern of one-sidedness applies, and explores how in current group decision-making systems one-sided problem definitions might consistently tend to be exploited in a way that is detrimental to collective well-being, as well as how a system of group decision-making meeting the requirements of a General Collective Intelligence solves the problem of one-sidedness to reliably maximize collective well-being.
\end{abstract}

\section{Introduction}

Problem definitions might be one-sided in taking into account one class of negative outcomes associated with the problem, at the same time as ignoring other negative outcomes associated with interventions that try to address the problem. While one-sidedness is generally perceived as negative, at the same time it is possible to envision scenarios in which one-sidedness might be the optimal response. An argument for such a scenario begins with the observation that human beings are believed to be capable of two kinds of thought, rational methodical (slow) reasoning, and intuitive (fast) reasoning [1]. Rational methodical reasoning essentially represents the reasoning process as an "equation" to be evaluated. Intuitive reasoning processes essentially come to a decision through estimation from past patterns. From the functional modeling perspective [2], both types of thought processes can be represented as having inputs, outputs, and a context of execution (in the case of rational methodical reasoning the context metaphorically represents the constants of the "equation" and other information aside from the values of the variables that are required to evaluate it).

Each individual uses both types of thought, but groups may consist of people who predominantly use one or the other in a given context, as some individuals might be predisposed to rational methodical reasoning in a given area, and some individuals might be predisposed to use intuitive reasoning [3]. The rational methodical reasoning group may attempt to use such "equations" even where the intuitive reasoning group might not believe those equations are representative of the problem, and therefore are not sensible, and certainly not "fair". While the intuitive reasoning group may reject the use of any such "equations" at all, even where the rational methodical reasoning group might believe those equations are highly representative of the problem, and therefore find the subjectivity that comes with avoiding use of such an equation "dishonest". 
Both reasoning styles are useful [4], [5], [6]. Intuitive reasoning is more highly associated with emotion, and emotion is a much more powerful mechanism for uniting individuals behind a movement than rational methodical reasoning. Because everyone who is moved by a single motivation communicated by a compelling speaker is moved in the same direction [7]. Relying on logic alone rather than relying on whatever motivation resonates with the crowd is far less effective. Logic is inherently divisive. Everyone might have a different reasoning process or "equation" they use to process logic, and everyone might perceive different "facts" to plug into those equations [8]. But where motivation is better for moving crowds in a single direction, logic is better at engineering tasks such as building bridges.

Regardless of whether each reasoning style might be more effective in a given domain of problem, each individual might not only tend towards a given reasoning style, but also might differ in the effectiveness with which they use that reasoning style. In essence one person (with the predisposition to the rational methodical approach) might tend to try to use an equation to get the answer, and the other person (with the predisposition to the intuitive approach) might tend to try estimates the answer. The person who uses the math equation might use the entirely wrong equation, they might use an equation that only addresses part of the problem, they might plug in the wrong values for the variables, they might evaluate the equation incorrectly, or they might commit some other error. Still, their approach is always to use an equation. So though the answers of one person using this reasoning style might tend to be accurate, the answers of another person using this reasoning style might tend to always be way off. Similarly, the person who uses their internal process for estimating answers from patterns perceived in the past (intuition) might view the world through unfounded perceptions, and therefore might have a very misdirected intuition. This situation is captured by the idiom "garbage in garbage out".

As mentioned, the intuitive reasoning style discounts the usefulness or applicability of such "equations", and therefore inherently discounts logical methodical reasoning that points to different conclusions than it has reached. And in this sense intuitive reasoning is by definition one-sided. But both one-sidedness and intuition are still essential. Because in any problem domains in which rational methodical reasoning is not possible but in which a conclusion still needs to be reached, intuition must be used. Such problem domains include those in which logical methodical reasoning is not available, or in which insufficient information, or insufficiently trustworthy information is available to evaluate that reasoning.

When a problem is new and all sides of the issue have not been sufficiently explored to develop a logical framework for understanding, or the truth of that understanding has not been sufficiently tested in order to define an "equation" with which to methodically guide reasoning, then it may be true that logical methodical reasoning is not available. Attempts to use this deductive reasoning must then fail in such cases. But the failure points of logical methodical reasoning do not extend to intuitive reasoning. A chain of logical reasoning excludes a great many possible solutions, particularly as the chain gets longer and more complex, since the outputs of one reasoning process must provide the inputs to the next reasoning process if logical jumps are to be avoided. And the final solution must align with all those component reasoning processes and their inputs and outputs. Not only does a long chain of reasoning potentially restrict the end result of that reasoning to just a few possibilities out of a potentially great many, but if any of the information required to evaluate that reasoning is missing (inputs, context), it is possible that no conclusion may be reached at all.

However, since any category of problem can potentially be related to any of the total number of categories of problems that exist in an individual cognition, and since any category of solution can 
potentially be related to any of the categories of solutions that exists as well, then the potential relationships that can be searched for patterns represented by past observations may result in a far larger number of potential conclusions. Where logical methodical reasoning might fail to make a conclusion at all, intuitive reasoning, being based on such patterns with so many solutions, then might reliably arrive at one. Whether or not that initial conclusion is correct, in a system of adaptive reasoning that is reliably capable of converging on the correct solution, any answer, even one that is eventually proven incorrect, still adds to the information with which the system might guide itself to the correct conclusion.

\section{The Case of the COVID-19 Response}

Overwhelmingly, news coverage of the COVID-19 response has often suggested that governments could and should do more to stop the spread of the virus in order to save lives, and by stating that considering economic impact over human lives is immoral, have implied that any failure to do so must stem from such an immoral motivation. In some cases on social media individuals have suggested that if politicians don't take whatever measures are being suggested then each death resulting from this new coronavirus will be essentially an act of murder they have committed [9]. Such rhetoric leaves little room for anything else other than over reaction. However, the economic shutdowns that have taken place around the world in response to the pandemic also have the potential to cost lives. Some of the researchers responsible for defining mathematical models of the potential spread of the pandemic and the potential mortality as a result, have openly voiced their opinions on the need for models representing the potential deaths resulting from the economic impact of the shutdowns mandated in response [10]. Although such models of the economic impact appear to exist [11] - [16], the fact remains that the public response has been either one sided in favor of shutdown measures that have been criticized as excessive with potentially dramatic economic consequences, or one-sided in favor of measures that have been criticized as insufficient and potentially leaving the population at risk. In general, governments have not been able to provide models justifying the exact scale of their response. Since all the information appears to exist, the question is why no current decision-systems appear to have the capacity to reliably converge on including all available information.

\section{Hedging for Risk in the Face of Uncertainty}

Another part of the problem is uncertainty. We don't know how long people will remain sick with COVID-19 and therefore unable to participate fully in the economy. We don't know if they will tend to develop immunity or tend to get reinfected again. In fact we don't know quite a number of factors that will determine the economic impact of the pandemic independently of any shutdown. For this reason a greater level of caution might be optimal than the most likely projections of mortality from the pandemic might warrant. Because it might be worthwhile for public health officials to hedge their bets. Still, some methodology is required in order to use all available information so bets can be hedged intelligently.

\section{Exploitation of the COVID-19 Problem by Centralized Interests}

The FMF suggests that in order to optimize collective outcomes for any group, centralized decisionmaking must be balanced with decentralized. Decentralization is required to maximize outcomes for all participants in any process so that all outcomes which achieve that maximization become available to the group. And centralization is required to prioritize the functions of certain participants in group processes where there outputs are higher, so that the value created for all participants is maximized. This balance between centralization and decentralization shifts dynamically in natural systems. For example, within the human body, in some circumstances blood circulation to the internal organs might be restricted to ensure adequate supply to the brain. And in other circumstances blood might be maximally distributed to the extremities to ensure all other functions are operating optimally. 
The FMF defines many layers of decentralization. In any decision-making system that lacks these many layers, the FMF suggests that decision-making will be centralized in that it will be aligned with the interests of a subset of decision-makers. Wherever centralization constrains decision-making in this way, game theory modeling suggests [17] that this constraint can potentially act as a hidden "tax" that extracts value from any interaction and allocates that value to the decision-maker.

One-sidedness might be essential for coming to an initial conclusion, but in refining that conclusion such bias might be a significant source of error [18] - [22], in particular if there is no objective standard for what action is "sufficient" as a result of such bias. If government responses to COVID-19 in the very politically polarized media environment [25] might tend to be constrained to eventually overreact in the absence of mathematical models for deaths that might be caused by measures to combat the pandemic, such as economic shutdowns, then by definition of the term "overreact" being "too much", these responses do not maximize collective well-being. Furthermore, if all decision-making systems have an imbalance of centralization in the absence of GCI as suggested by the FMF, and if this centralization of decision-making tends to align outcomes with the interests of a subset of decisionmakers, this suggests that rather than converging over time on interventions that maximize collective well-being, due to this potential opportunity for extracting value towards their interests, departures from optimum collective well-being may actually be driven by those same centralized interests.

\section{The Generalized Case}

Modeling reasoning processes as solutions to cognitive problems, in the generalized case, individual minds (systems of individual cognition) have a single framework for comparing solutions (comparing conclusions from any different reasoning processes). This framework is defined by the impact on the individual's well-being. In the general case for groups, a problem has a projected impact on the group over some time span, as does each prospective intervention. In order to be comparable, these impacts must be related through some universal framework. The FMF suggests that the only framework universal enough to enable this general comparison, and therefore general enough to enable general problem solving ability, is provided by an assessment of collective well-being.

For example, if anticipated weapons of mass destruction can potentially kill 1 million Americans or American allies with a $2 \%$ probability of being deployed, how does that compare to a war that costs the US taxpayer X dollars that could potentially contribute to their collective well-being if allocated elsewhere? If all potential interventions and potential outcomes could be added to such a model, and if that model were deployed within a GCI with the capacity to adapt to better solve the problem, that model could become more "intelligent" over time.

Perception is also not equal for all risks or for all potential rewards. A $1 \%$ chance of getting dismembered and devoured alive by wild dogs while enjoying a pleasant hike might warrant more attention than a $1 \%$ chance of dying from an overdose of some drug during recreational drug use, or a $1 \%$ chance of dying from the bends after an exhilarating scuba dive. The fear of being eaten by wild dogs may cause all wild dogs to be exterminated from all urban environments. However the lesser fear of death by overdose may not cause individuals to eliminate all contact with such substances. And the lesser fear of death from the bends may not cause individuals to stop diving. The effort put into eliminating highly perceived risks, or put into achieving highly perceived benefits, might for this reason be a significant multiple of efforts put into eliminating low perceived risks or low perceived benefits [23], [24].

However, a critical issue with this approach is that decentralizing decision-making requires providing widespread access to information. And just like some thoughts are too painful for an individual to 
admit to themselves, leading to self-deceiving false justifications, some reasoning processes that are too unacceptable to some members of a group might only be used covertly if they aren't to provoke a negative reaction that makes their use impossible. And without GCI to define a single "collective wellbeing" that might be improved by reconciling such actual motivations with expressed intentions, the natural balance might as a result be the tendency for subsets of the group who are driven by such reasoning to hide their motivations. Therefore there may be forces in groups that might act to keep subsets of reasoning outside the collective intelligence process, while at the same time preventing the collective intelligence from improving it's problem solving ability (i.e. becoming more intelligent) through the inclusion of those processes.

\section{Conclusions}

In terms of the big five personality traits, for an individual mind, the issue of one-sidedness is rarely the absence of information, but instead might be the result of a lack of openness to information that supports a contrary viewpoint, a lack of conscientiousness in reviewing all available information, and potentially a high level of agreeableness with proponents of popular opinions rather than the tendency to search for holes in logic that provide the opportunity to disagree. For groups, the issue of onesidedness is also influenced by the fact that without GCI groups may be constrained to act as a collection of individuals rather than a single collective entity with a collective collective well-being that is well-defined and coherent enough to drive group reasoning. GCI is defined as a system of decisionmaking system capable of becoming more fit in problem-solving through various domains of adaptation. Of particular importance to the issue of one-sidedness is the domain of adaptation by cooperation that all models of GCI as defined by the FMF must have. This domain of cooperation ensures that all individuals in the collective cooperate to share information and reasoning in a way that converges on whatever understanding maximizes collective outcomes rather than converging on whatever understanding maximizes outcomes for the most powerful subset of decision-makers. If intelligence, or general problem solving ability, maximizes impact on the general problem of wellbeing, then by definition, such an understanding is the most intelligent one.

\section{References}

[1] Kahneman, D. (2012).Thinking, fast and slow.London: Penguin Books. Isbn: 0141033576.

[2] Andy E. Williams, A Functional Modeling Framework for Representing and Comparing Models of Consciousness or Cognition, under review

[3] Thorsten Pachur, Melanie Spaar, Domain-specific preferences for intuition and deliberation in decision making, Journal of Applied Research in Memory and Cognition, Volume 4, Issue 3, 2015, Pages 303-311, ISSN 2211-3681, https://doi.org/10.1016/j.jarmac.2015.07.006.

[4] Farah Focquaert, Megan S. Steven, George L. Wolford, Albina Colden, Michael S. Gazzaniga, Empathizing and systemizing cognitive traits in the sciences and humanities, Personality and Individual Differences, Volume 43, Issue 3, 2007, Pages 619-625, ISSN 0191-8869, https://doi.org/10.1016/j.paid.2007.01.004.

[5] Mark Brosnan, Chris Ashwin \& Tim Gamble (2013) Greater Empathizing and reduced Systemizing in people who show a jumping to conclusions bias in the general population: Implications for psychosis, Psychosis, 5:1, 71-81, DOI: 10.1080/17522439.2011.626526

[6] Mark Brosnan, Melissa Hollinworth, Konstantina Antoniadou, Marcus Lewton, Is Empathizing intuitive and Systemizing deliberative?, Personality and Individual Differences, Volume 66, 2014, Pages 39-43, ISSN 0191-8869, https://doi.org/10.1016/j.paid.2014.03.006.

[7] Thagard, P., Kroon, F.W. Emotional consensus in group decision making. Mind \& Society 5, 85104 (2006). https://doi.org/10.1007/s11299-006-0011-5

[8] Miller, B. When is consensus knowledge based? Distinguishing shared knowledge from mere agreement. Synthese 190, 1293-1316 (2013). https://doi.org/10.1007/s11229-012-0225-5 
[9] (2020) IS DONALD TRUMP CRIMINALLY RESPONSIBLE FOR CORONAVIRUS DEATHS?, April 2 2020, 6:15 a.m [theintercept.com]. Available at URL: https://heintercept.com/2020/04/02/isdonald-trump-criminally-responsible-for-coronavirus-deaths

[10] Martin Enserink, Kai Kupferschmidt, Mathematics of life and death: How disease models shape national shutdowns and other pandemic policies, Published Mar. 25, 2020, 6:40 PM, Accessed Mar. 30, 2020 from: https:/www.sciencemag.org/news/2020/03/mathematics-life-and-death-how-diseasemodels-shape-national-shutdowns-and-other\#

[11] Ruhm, C. J. ( 2016) Health Effects of Economic Crises. Health Econ., 25: 6- 24. doi:

$10.1002 /$ hec. 3373 .

[12] Gerard J. van den Berg, Alexander Paul and Steffen Reinhold, Economic conditions and the health of newborns: Evidence from comprehensive register data, Labour Economics,

10.1016/j.labeco.2020.101795, (101795), (2020).

[13] Kristín Helga Birgisdóttir, Arna Hauksdóttir, Christopher Ruhm, Unnur Anna Valdimarsdóttir and Tinna Laufey Ásgeirsdóttir, The effect of the economic collapse in Iceland on the probability of cardiovascular events, Economics \& Human Biology, 10.1016/j.ehb.2020.100861, (100861), (2020). [14] Spyros Makridakis, Robin M. Hogarth, Anil Gaba, Forecasting and uncertainty in the economic and business world, International Journal of Forecasting, Volume 25, Issue 4, 2009, Pages 794-812, ISSN 0169-2070, https://doi.org/10.1016/j.ijforecast.2009.05.012.

[15] Bohk, C., \& Rau, R. (2015). Impact of Economic Conditions and Crises on Mortality and its Predictability. Kolner Zeitschrift fur Soziologie und Sozialpsychologie, 67(Suppl 1), 271-294. https://doi.org/10.1007/s11577-015-0323-8

[16] Tapia Granados, JA, Ionides, EL. Population health and the economy: Mortality and the Great Recession in Europe. Health Economics. 2017; 26: e219- e235. https://doi.org/10.1002/hec.3495 [17] Andy E. Williams, The Principles Enabling Cooperation within Conscious Organisms and Collectives, working paper

[18] Brenner, L.A., Koehler, D.J. and Tversky, A. (1996), On the evaluation of one-sided evidence. J. Behav. Decis. Making, 9: 59-70. doi:10.1002/(SICI)1099-0771(199603)9:1<59::AID-

BDM216>3.0.CO;2-V

[19] Daniel C. Feiler, Jordan D. Tong and Richard P. Larrick, Biased Judgment in Censored

Environments, Management Science, 10.1287/mnsc.1120.1612, 59, 3, (573-591), (2013).

[20] Walton, D (1999) One-sided Arguments: A Dialectical Analysis of Bias. New York: SUNY Press. Google Scholar

[21] Keith E. Stanovich \& Richard F. West (2008) On the failure of cognitive ability to predict myside and one-sided thinking biases, Thinking \& Reasoning, 14:2, 129-167, DOI:

$10.1080 / 13546780701679764$

[22] Stanovich, K. E., West, R. F., \& Toplak, M. E. (2013). Myside Bias, Rational Thinking, and Intelligence. Current Directions in Psychological Science, 22(4), 259-264.

https://doi.org/10.1177/0963721413480174

[23] Fabio Del Missier, Timo Mäntylä and Wändi Bruine Bruin, Decision-making Competence, Executive Functioning, and General Cognitive Abilities, Journal of Behavioral Decision Making, 25, 4, (331-351), (2011).

[24] Jessen L. Hobson and Steven J. Kachelmeier, Strategic Disclosure of Risky Prospects: A Laboratory Experiment, The Accounting Review, 10.2308/accr.2005.80.3.825, 80, 3, (825-846), (2005). [25] Martin Gilens and Craig Hertzman, "Corporate Ownership and News Bias: Newspaper Coverage of the 1996 Telecommunications Act," The Journal of Politics 62, no. 2 (May, 2000): 369-386. https://doi.org/10.1111/0022-3816.00017 